\title{
Lopinavir-Ritonavir in the Treatment of COVID-19: A Dynamic Systematic Benefit-Risk Assessment
}

\author{
Vicki Osborne $e^{1,2} \cdot$ Miranda Davies $^{1,2} \cdot$ Samantha Lane $e^{1,2} \cdot$ Alison Evans $^{1,2} \cdot$ Jacqueline Denyer $^{1} \cdot$ \\ Sandeep Dhanda ${ }^{1,2} \cdot$ Debabrata Roy $^{1,2} \cdot$ Saad Shakir ${ }^{1,2}$
}

Published online: 23 June 2020

(c) The Author(s) 2020

\begin{abstract}
Introduction and Objective COVID-19 is an ongoing, global public health crisis for which safe and effective treatments need to be identified. The benefit-risk balance for the use of lopinavir-ritonavir in COVID-19 needs to be monitored on an ongoing basis, therefore a systematic benefit-risk assessment was designed and conducted. A key objective of this study was to provide a platform for a dynamic systematic benefit-risk evaluation; although initially this evaluation is likely to contain limited information, it is required because of the urgent unmet public need. Importantly, it allows additional data to be incorporated as they become available, and re-evaluation of the benefit-risk profile.

Methods A systematic benefit-risk assessment was conducted using the Benefit-Risk Action Team (BRAT) framework. The exposure of interest was lopinavir-ritonavir treatment in severe COVID-19 compared to standard of care, placebo or other treatments. A literature search was conducted in PubMed and Embase to identify peer-reviewed papers reporting clinical outcomes. Two clinicians constructed a value tree and ranked key benefits and risks in order of considered clinical importance. Results We screened 143 papers from PubMed and 264 papers from Embase for lopinavir-ritonavir; seven papers were included in the final benefit-risk assessment. In comparison to standard of care, data for several key benefits and risks were identified for lopinavir-ritonavir. Time to clinical improvement was not significantly different for lopinavir-ritonavir in comparison to standard of care (hazard ratio 1.31, 95\% confidence interval 0.95-1.80). From one study, there were fewer cases of acute respiratory distress syndrome with lopinavir-ritonavir compared with standard of care (13\% vs $27 \%)$. There also appeared to be fewer serious adverse events with lopinavir-ritonavir (20\%) vs standard of care (32\%). Limited data were available for comparison of lopinavir-ritonavir to other treatments.

Conclusions Based on currently available data, there was no clear benefit for the use of lopinavir-ritonavir compared to standard of care in severe COVID-19. Risk data suggested a possible decrease in serious adverse events. There was a reduction in acute respiratory distress syndrome with lopinavir-ritonavir in one study. Overall, the benefit-risk profile for lopinavirritonavir in severe COVID-19 cannot be considered positive until further efficacy and effectiveness data become available.
\end{abstract}

Vicki Osborne

vicki.osborne@dsru.org

1 Drug Safety Research Unit, Bursledon Hall, Blundell Lane, Southampton SO31 1AA, UK

2 School of Pharmacy and Biomedical Sciences, University of Portsmouth, Portsmouth, UK

\section{Key Points}

COVID-19 is a global pandemic, for which lopinavirritonavir has been proposed as a possible treatment.

Based on currently available data, there is no clear benefit for the use of lopinavir-ritonavir compared to standard of care in severe COVID-19.

Ongoing clinical trial data can be incorporated into the framework when available to provide an updated benefitrisk assessment. 


\section{Introduction}

Coronaviruses have circulated in human and animal populations for many years and in humans they are a cause of respiratory tract infections [1]. More recently, severe acute respiratory syndrome coronavirus 2 emerged in Wuhan, China in December 2019 [2, 3]. Severe acute respiratory syndrome coronavirus 2 causes coronavirus disease (COVID-19) and this outbreak was declared a global pandemic by the World Health Organization in March 2020 [4]. Fever, cough and shortness of breath are the main reported symptoms of COVID-19 [5] but this disease also has a concerning case mortality rate among certain populations, such as older adults and those with underlying health conditions.

In the current COVID-19 pandemic, there is a need to identify effective safe treatments as rapidly as possible. Lopinavir-ritonavir (LPVr) is a combination protease inhibitor and nucleoside analogue used for the treatment of human immunodeficiency virus (HIV) type 1 [6]. The use of LPVr in severe acute respiratory syndrome has been examined previously and indicated a favourable clinical response, with a decreased incidence of adverse clinical outcomes at day 21 , reduced viral load at $48 \mathrm{~h}$, decreased steroid use and reduced incidence of nosocomial infections [7]. For this reason, multiple trials in COVID-19 are currently being conducted to determine if LPVr is an effective treatment, including the worldwide RECOVERY trial and the World Health Organization's SOLIDARITY trial [8-10]. It is essential to examine the benefit-risk profile of all medications, but ongoing monitoring is especially important where treatments may be used with limited evidence in new indications. Lopinavir-ritonavir is already being used as a standard treatment for COVID-19 in some countries. Systematic reviews have been conducted to examine the efficacy of LPVr in the treatment of COVID19 , though not all of these have been peer reviewed to date [11]; so far these have concluded that there is a lack of evidence of efficacy $[11,12]$. However, a systematic benefit-risk assessment on the use of LPVr for COVID-19 treatment, based on currently available evidence, has not yet been conducted.

The Benefit-Risk Action Team (BRAT) uses a structured descriptive framework to outline the key benefits and risks of a medication within a defined disease context. If sufficient relevant data are available, an additional quantitative assessment can be used to further examine the benefit-risk profile [13]. The BRAT was also designed to assist communication with regulatory authorities [14]. The framework design allows for transparency in the decisionmaking process and assumptions can be explored further by sensitivity analyses using quantitative methods [15].
This dynamic approach to a benefit-risk assessment of potential treatments for COVID-19 has been previously applied to the anti-viral agent remdesivir [16].

The systematic benefit-risk assessment for LPVr was conducted based on publicly available data to 13 May, 2020. Because of continuous emerging data on the use of $\mathrm{LPVr}$ in COVID-19, the framework can be subsequently used to repeat the assessment as further data arise, allowing for rapid and dynamic evidence-based decision making as more relevant data become available. This assessment was conducted to aid clinicians and public health and regulatory authorities in the decision-making process on treatments for COVID-19.

\section{Objectives}

This study aimed to examine the benefit-risk profile of $\mathrm{LPVr}$ in patients with COVID-19 compared to standard of care, placebo or other treatments. A key objective of this study was to provide a platform for a dynamic systematic benefitrisk evaluation; although initially this evaluation is likely to contain limited information, it is required because of the urgent unmet public need. Importantly, it allows additional data to be incorporated as they become available, and reevaluation of the benefit-risk profile.

\section{Methods}

\subsection{Benefit-Risk Framework}

The BRAT framework was used to assess the overall benefitrisk of using LPVr as a treatment for COVID-19 compared to standard of care, placebo or other treatments. The BRAT uses a six-step iterative process to support the decision and communication of a benefit-risk assessment: define decision context, identify outcomes, identify data sources, customise framework, assess outcome importance, and display and interpret key benefit-risk metrics [17]. Three settings of interest for use of COVID-19 treatments were identified; treatment for severe disease, treatment of mild disease in the community and prevention in healthcare professionals exposed to the virus. This assessment focuses on $\mathrm{LPVr}$ for the treatment of severe COVID-19 disease, which we defined as hospitalisation as a result of COVID-19 infection.

\subsubsection{Population of Interest}

Patients with COVID-19 who were treated with LPVr were the population of interest, while patients receiving standard of care, placebo or other treatments were the comparators of interest. Where standard of care was reported as the comparator group, this refers to no use of a specific pharmaceutical 
treatment for COVID-19 but provision of standard supportive therapy within the hospital setting.

\subsubsection{Outcomes of Interest}

All potential benefits and risks related to $\mathrm{LPVr}$ were initially identified, regardless of their importance. Key benefits and risks were selected by clinician judgement, i.e. those considered to be of clinical importance or potentially serious, and included in the value tree, which provides a visual representation of these outcomes in the context of severe COVID-19 disease. These benefits and risks displayed in the value tree were ranked according to perceived importance (benefits) and potential seriousness (risks). Two clinicians performed this ranking after discussion of the importance of each benefit and risk. Risks were categorised according to which system organ class (SOC) they belonged to, and where multiple events were identified within the same SOC, the ranking was based on the most serious event(s) within that SOC, with the most serious event(s) in each SOC presented first.

\subsubsection{Data Sources and Customisation of the Framework}

A literature search was performed in PubMed and Embase using the following search strategy: (lopinavir AND ritonavir) AND (covid* OR SARS-CoV-2 OR n?CoV OR coronavirus). Two reviewers examined articles for eligibility and a third clinical reviewer made the final decision on inclusion. Papers were included in the data extraction tables if they reported quantitative data on effectiveness and/or safety of LPVr in patients with severe COVID-19. Case reports and case series were excluded from the data extraction tables, but were considered for construction of the value tree. Results were restricted to English language only (abstracts in English language were acceptable where sufficient data provided) and peer-reviewed publications from 2019 to 13 May, 2020. Data were extracted for each benefit and risk, for LPVr and the comparator (standard of care, placebo or other treatments), where available. EudraVigilance spontaneous reporting data (up to 8 May, 2020) for LPVr where used in COVID-19 were also examined. EudraVigilance data were examined because of the usefulness of identifying real-world reports of adverse events outside of the clinical trial setting and because the publicly available version of this specific data source is frequently updated. After data extraction, a second reviewer performed a quality-control check on the extracted data.

\subsection{Outcome Assessment}

Key benefits and risks associated with the use of LPVr were identified for inclusion in the value tree from available data sources, including the product information, regulatory assessment reports and published literature. Predicted key benefits (clinical endpoints) were derived from both the published literature and in the case of ongoing studies, available clinical trial protocols. These benefits and risks, as presented in the value tree, were derived from both qualitative and quantitative data. A summary benefit-risk table was created to allow visualisation of the magnitude of each benefit and risk. Risk differences and corresponding 95\% confidence intervals (CIs) were calculated for each outcome where both numerator (number of events) and denominator (number of patients at risk) were available for both the treatment group (LPVr) and comparator group. No appropriate comparator groups were identified for LPVr within EudraVigilance owing to the recent nature of the pandemic and the lack of treatments made available for COVID-19 outside of the clinical trial setting. As such, adverse events reported for a comparison treatment in EudraVigilance were not available. Consequently, spontaneous reports are not included in the benefit-risk table and are presented in the text only.

Given the limited data at this point in time, further quantitative assessment was not considered appropriate. However, such methods can be used to account for both the importance of outcomes and the size of the study from which data were extracted by weighting outcomes. In anticipation of using further quantitative methods in the future when further data are made available, we have ranked the outcomes in the value tree by considered clinical importance.

\section{Results}

Figure 1 displays the value tree of the key benefits and risks related to LPVr treatment in COVID-19, which was constructed using a wide variety of data sources.

\subsection{Benefits}

Key benefits have been listed in the value tree in descending order of perceived clinical importance. At the current time, only one clinical trial was identified that provided empirical data for any of the clinical endpoints listed in the value tree [18]. Whilst the primary objective of this clinical trial was time to clinical improvement, additional data were provided for various endpoints including mortality risk, risk and duration of invasive mechanical ventilation, risk of non-invasive ventilation and oxygen requirement.

\subsection{Risks}

Key risks were identified for LPVr based on the current available evidence. It is acknowledged that this product is not licenced for use in the treatment of COVID-19 disease, and whilst safety data are available for its licenced use in 


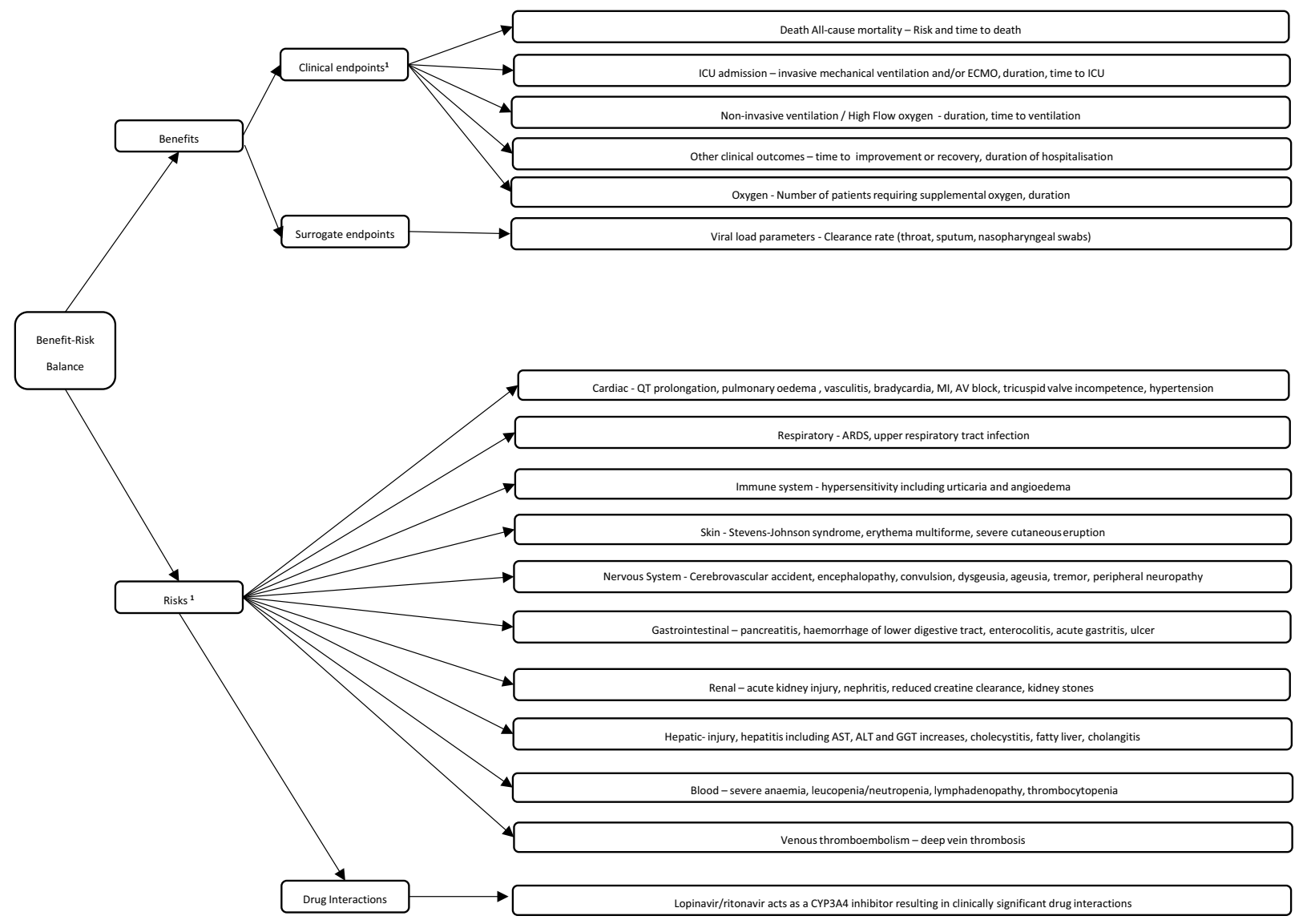

'Both benefits and risks have been ranked according to perceived clinical significance. Risks have been categorised according to system organ class (soc). The Socs have been ranked based on the most serious events//vents within each system, which have

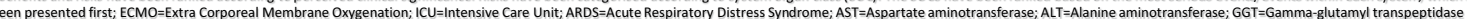

Fig. 1 Value tree of key benefits and risks identified for lopinavir/ ritonavir, ranked by order of clinical significance. Superscript 1: both benefits and risks have been ranked according to perceived clinical significance. Risks have been categorised according to system organ class. The system organ classes have been ranked based on the most

HIV type 1, its safety profile in the context of COVID-19 is largely unknown. Furthermore, for the limited safety data that are available for its use in COVID-19, it is often unclear whether the reported adverse event is due to the use of LPVr, or attributable to the underlying disease. Potentially serious risks that are likely to still pose a risk with the proposed short-term use of LPVr for COVID-19 have been summarised in the value tree and ranked according to perceived seriousness.

One of the most serious risks is prolongation of the QT interval, and the subsequent increased risk of sudden cardiac death [19-22]. Patients with COVID-19 are already predisposed to the development of cardiac arrhythmia owing to the effect of the virus on metabolic dysfunction, myocardial inflammation and the sympathetic nervous system serious events/events within each system, which have been presented first. $A L T$ alanine aminotransferase, $A R D S$ acute respiratory distress syndrome, AST aspartate aminotransferase, CYP cytochrome P450, $E C M O$ extracorporeal membrane oxygenation, $G G T$ gamma-glutamyl transpeptidase, $I C U$ intensive care unit, $M I$ myocardial infarction

[20]. Additionally, it is important to note that LPVr is an inhibitor of cytochrome P450 3A4, and therefore it cannot be used with other medicines that are substrates of this enzyme, such as chloroquine, which itself can cause QT prolongation [21]. In addition to the effects on QT prolongation, $\mathrm{LPVr}$ has also been shown to cause modest asymptomatic prolongation of the PR interval in some healthy adult subjects, with rare reports of second- or third-degree atrioventricular block in patients with underlying structural heart disease and pre-existing conduction system abnormalities or in patients receiving drugs known to prolong the PR interval (such as verapamil or atazanavir), and therefore LPVr should be used with caution [23] in such patients.

Lopinavir/ritonavir are both inhibitors of the cytochrome $\mathrm{P} 4503 \mathrm{~A}$ isoform, and therefore treatment is likely to 
increase plasma concentrations of concomitant medicinal products that are primarily metabolised by cytochrome $\mathrm{P} 450$ 3A [23]. Clinically significant drug interactions have been observed with LPVr use during treatment for COVID-19, including increased plasma concentrations of direct oral anticoagulants [24], and increased plasma concentrations of immunosuppressants in organ transplant recipients [25].

Certain risks factors and clinical characteristics have been associated with the development of acute respiratory distress syndrome (ARDS) amongst patients with COVID-19 [26]. Patients who developed COVID-19-related ARDS were likely to require admission to the intensive care unit. In the study by Cao et al., respiratory failure/ARDS was reported as a serious adverse event in both treatment groups [18]. Whilst causality in these cases is not known, it would seem likely that these cases were attributable to progression of the underlying COVID-19 disease.

Hypersensitivity reactions such as urticaria and angioedema are reported to occur commonly with the use of LPVr for the treatment of HIV, and rarely serious skin reactions such as Stevens-Johnson syndrome and erythema multiforme have been reported with its use in this treatment population [23]. Gastrointestinal side effects of LPVr are well recognised, and diarrhoea and nausea are very common. Serious gastrointestinal adverse effects included in the key risks for this assessment include pancreatitis, which has been associated with the use of LPVr [7, 23, 27]; most patients who developed pancreatitis during treatment for HIV had a prior history of this condition. Treatment with LPVr has been associated with an increase in triglycerides in patients treated for HIV [23], and amongst patients treated for COVID-19 [28-31], which is likely to be another contributory factor in the development of pancreatitis.

In the context of treatment for HIV, LPVr has been uncommonly associated with certain adverse renal outcomes, including a reduction in creatine clearance, nephritis and haematuria [23]. Cases of acute kidney injury have been reported in patients taking LPVr in COVID-19; however, it is unclear whether there is any association, as this outcome was reported more frequently amongst patients in the standard of care comparator group [18] in addition to overall limited safety data availability. Elevations of liver enzymes have also been commonly reported with the use of LPVr in the treatment of HIV [23], and liver injury has been reported in patients treated with LPVr for COVID-19 [32, 33]. Blood dyscrasias have also been associated with the use of LPVr during HIV treatment [23], with reports of severe anaemia amongst patients treated for COVID-19 [18].

\subsection{Quantitative Data}

Data for outcomes are presented in the data extraction table and key benefit-risk summary table (Tables 1 and 2, respectively). From literature searching, we identified 143 papers from PubMed and 264 papers from Embase for LPVr. All papers were reviewed for information on the benefits and risks relating to LPVr, including both quantitative and qualitative data. After initial review and removal of duplicates, 43 papers were identified with information on safety of LPVr and 15 papers with efficacy information for LPVr. These papers were reviewed further to determine whether they met all inclusion criteria; seven papers were included in the final benefit-risk assessment.

In comparison to standard of care, data for several key benefits and risks for LPVr were identified. In the Cao et al. trial [18], the benefit of time to clinical improvement (intention-to-treat analysis) was not statistically significant after adjustment for other covariates in comparison to standard of care ( 16 vs 16 days, hazard ratio $=1.31,95 \%$ CI $0.95-1.80$ ). Other non-significant benefit data were identified in this trial including median duration of mechanical ventilation (risk difference $=-1$ day, $95 \% \mathrm{CI}-4$ to 2 ) and death at 28 days (risk difference $=-0.06,95 \% \mathrm{CI}-0.17$ to 0.06 ) .

Risk data were mainly available from the Cao et al. trial, which reported fewer serious adverse events in patients taking LPVr (20\%) compared to standard of care (32\%). There were fewer cases of ARDS with LPVr compared to standard of care (13\% vs $27 \%)$. The difference in the proportion of adverse events between groups was not tested statistically in the study and the study was also not powered to detect differences in adverse events.

Limited data were available for comparison of LPVr to other treatments. Viral clearance at day 14 for LPVr was lower compared with arbidol (85\% vs $91 \%$ ), and there was minimal difference in mean time to viral clearance (10.20 days vs 10.11 days, respectively). Data were only available for one risk in comparison to arbidol; a lower proportion of those patients treated with LPVr experienced increased ALT compared with arbidol (9\% vs 19\%, respectively).

In spontaneous reports from Eudravigilance, for $\mathrm{LPVr}$ used in COVID-19, there were 28 reports of hepatocellular injury, 15 reports of acute kidney injury, 17 reports of prolongation of the QT interval, six reports of ARDS and three reports of pancreatitis. Information on case reports was only available from the publicly available dataset and thus was limited; however, these data are useful in identifying real-world reports of adverse events outside of the clinical trial setting.

\section{Discussion}

The study aim was to examine the benefit-risk profile of LPVr in COVID-19 patients compared to standard of care, placebo or other treatments. A key objective of this study 


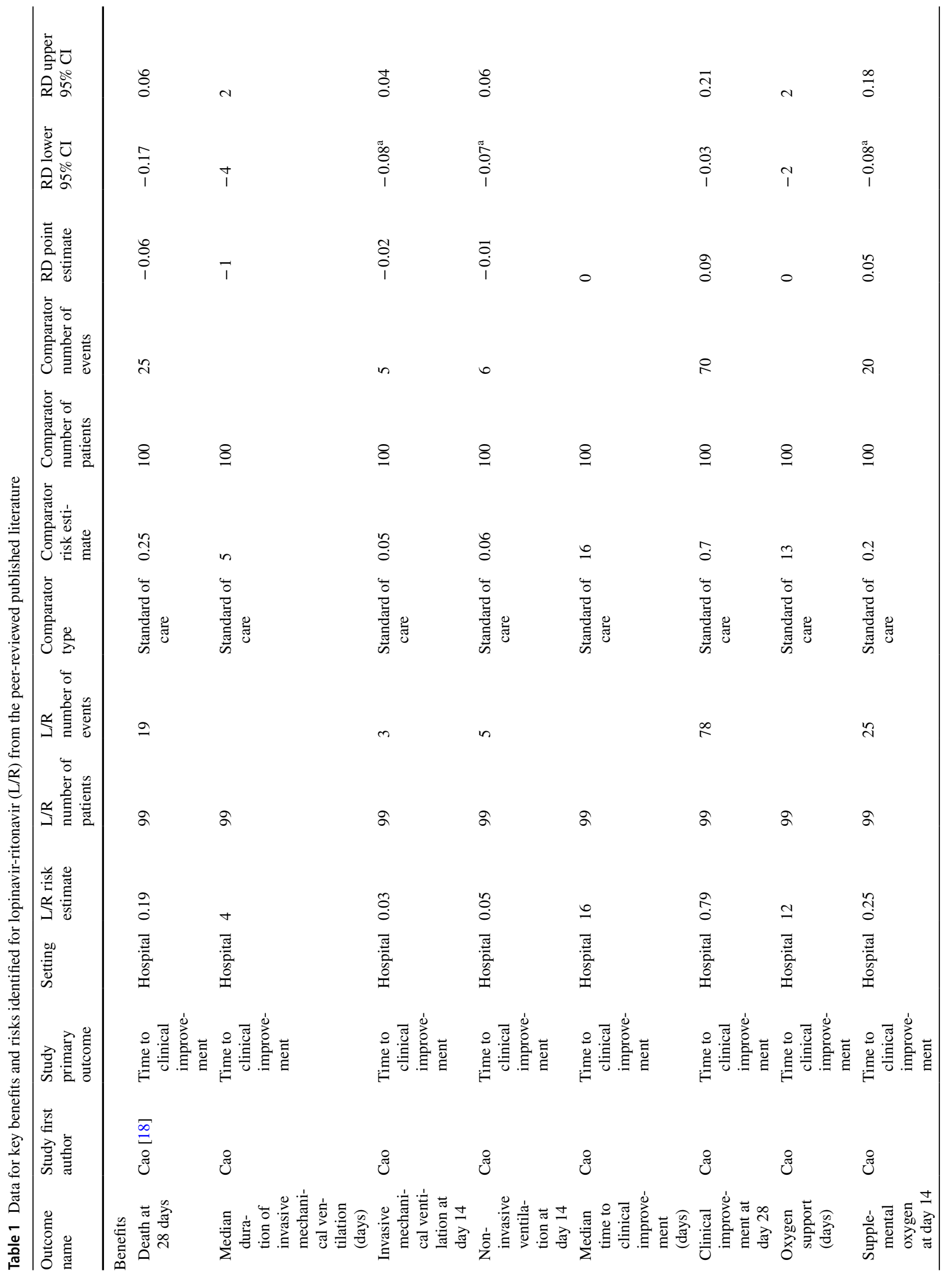




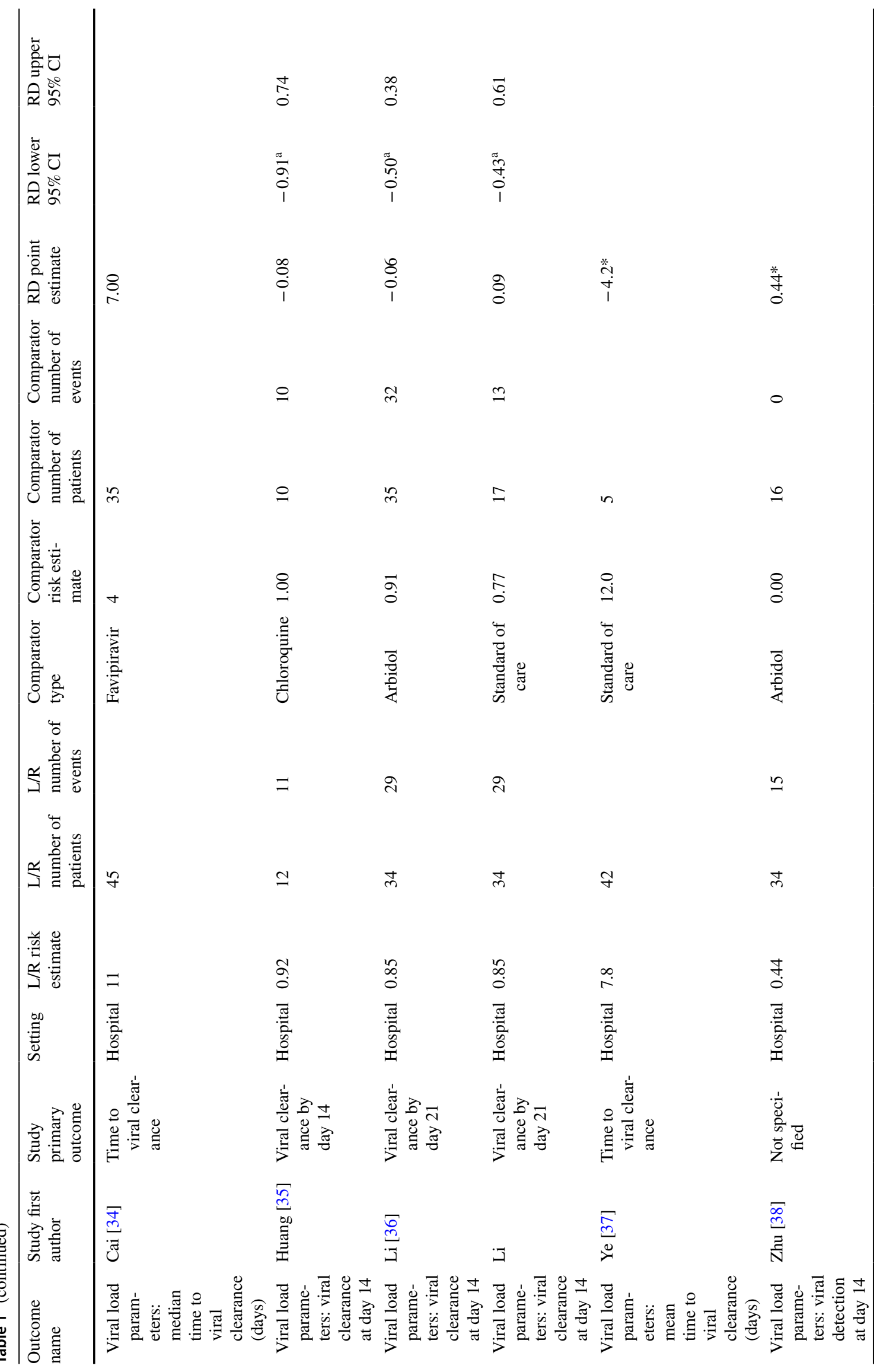




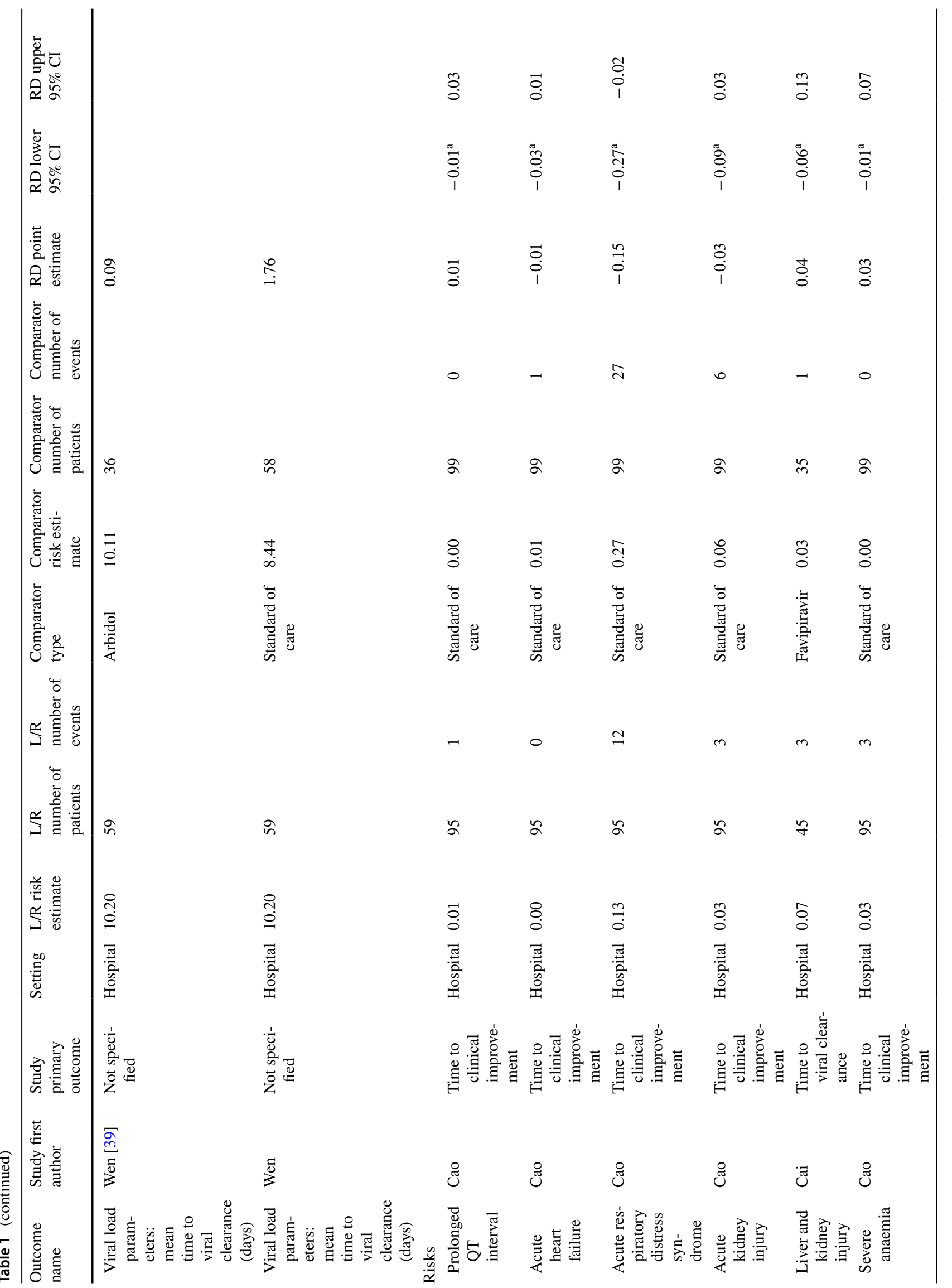




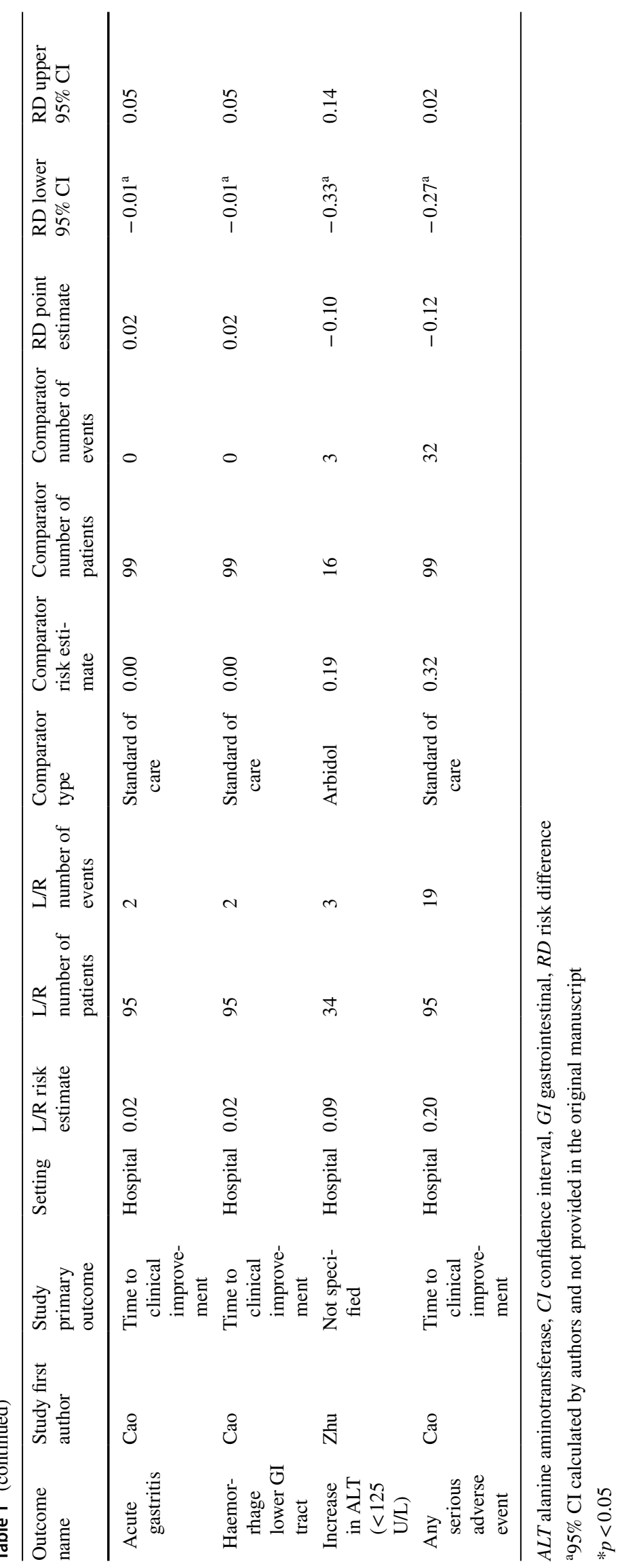


Table 2 Benefit-risk summary table for key benefits and risks identified for lopinavir-ritonavir (L/R) compared to standard of care

\begin{tabular}{|c|c|c|c|c|}
\hline Outcome name & $\begin{array}{l}\mathrm{L} / \mathrm{R} \text { risk/1000 } \\
\text { patients }\end{array}$ & $\begin{array}{l}\text { Standard of care } \\
\text { risk } / 1000 \text { patients }\end{array}$ & RD $(95 \% \mathrm{CI}) / 1000$ patients & Hazard ratio $(95 \% \mathrm{CI})$ \\
\hline \multicolumn{5}{|l|}{ Benefits } \\
\hline Death at 28 days & 192 & 250 & $-58(-173,57)$ & \\
\hline Invasive mechanical ventilation at day 14 & 30 & 50 & $-20(-75,36)^{\mathrm{a}}$ & \\
\hline Non-invasive ventilation at day 14 & 51 & 60 & $-9(-75,56)^{\mathrm{a}}$ & \\
\hline Time to clinical improvement & & & & $1.31(0.95,1.80)$ \\
\hline Clinical improvement at day 28 & 788 & 700 & $88(-33,209)$ & \\
\hline Supplemental oxygen at day 14 & 253 & 200 & $53(-80,185)^{\mathrm{a}}$ & \\
\hline Viral load parameters: viral clearance at day 14 & 853 & 765 & $88(-431,607)$ & \\
\hline \multicolumn{5}{|l|}{ Risks } \\
\hline Prolonged QT interval & 11 & 0 & $11(-10,31)^{\mathrm{a}}$ & \\
\hline Acute heart failure & 0 & 10 & $-10(-30,10)^{\mathrm{a}}$ & \\
\hline Acute respiratory distress syndrome & 126 & 273 & $-147(-272,-21)$ & \\
\hline Acute kidney injury & 32 & 61 & $-29(-89,31)^{\mathrm{a}}$ & \\
\hline Severe anaemia & 32 & 0 & $32(-4,67)^{\mathrm{a}}$ & \\
\hline Acute gastritis & 21 & 0 & $21(-8,50)^{\mathrm{a}}$ & \\
\hline Haemorrhage lower GI tract & 21 & 0 & $21(-8,50)^{\mathrm{a}}$ & \\
\hline Any serious adverse event & 200 & 323 & $-123(-267,20)^{\mathrm{a}}$ & \\
\hline
\end{tabular}

$C I$ confidence interval, $G I$ gastrointestinal, $R D$ risk difference

${ }^{\mathrm{a}} 95 \% \mathrm{CI}$ calculated by authors and not provided in the original manuscript

was to provide a platform for a dynamic systematic benefitrisk evaluation; although initially this evaluation is likely to contain limited information, it is required because of the urgent unmet public need. Importantly, it allows additional data to be incorporated as they become available, and reevaluation of the benefit-risk profile. This paper provides a systematic benefit-risk assessment using the BRAT methodology and is inclusive of the available literature up to and including 13 May, 2020. Therefore, this represents a snapshot of the data available to date and outlines a clear and transparent framework into which subsequent clinical trial and observational study data can be incorporated, and the benefit-risk profile re-assessed.

At the current time, data relating to the benefits of LPVr are limited, with efficacy data for clinical endpoints available from only one published clinical trial. This trial found no statistically significant difference in the primary outcome of time to improvement (intention-to-treat analysis) between the two groups (LPVr treatment in addition to standard supportive care vs standard care alone); however, the sample size was small. There was some evidence (though not statistically significant) that LPVr reduced mortality at 28 days (95\% CI $19.2 \%$ vs $25.0 \%$ ), difference of $-5.8 \%$ (95\% CI -17.3 to 5.7). It is of note that the median time interval between symptom onset and randomisation was 13 days (interquartile range, 11-16 days), therefore it is unknown whether more favourable results may have been seen if drug treatment had been initiated earlier in the course of the disease.
The safety profile of LPVr in the treatment of severe COVID-19 disease is largely unknown. We identified key risks from its usage in the treatment of HIV, in addition to the limited safety data available from its use in COVID-19. Comparator safety data revealed a lower incidence of all serious adverse events amongst patients receiving LPVr compared with standard of care (risk difference - 123 events per 1000 patients). Very small numbers of cases of some adverse events were reported in the study by Cao et al., including severe anaemia, acute gastritis and lower gastrointestinal bleeding, all of which were only reported amongst patients taking LPVr. The incidence of acute kidney injury was lower in the Cao et al. trial amongst patients taking LPVr compared with standard of care (3\% vs $6 \%$ ). The incidence of ARDS was also observed to be lower in patients using LPVr compared with standard of care (13\% vs $27 \%)$. It is acknowledged that ARDS is a known outcome in COVID-19 and thus a reduction in risk could potentially be considered a benefit of LPVr treatment. Acute respiratory distress syndrome is included as a risk in the current value tree because it was considered an adverse event in all trials examined; however, the benefit-risk assessment may be updated if sufficient evidence becomes available to suggest it should be considered a benefit instead. Consequently, the incidence of ARDS with LPVr in COVID-19 should be intensively monitored as further data are made available to determine if this could potentially be a benefit of treatment. 
Overall, there was a lack of efficacy data with no clear benefits identified for LPVr treatment compared with standard of care. Risk data, although limited, suggested a possible decrease in adverse events for some serious outcomes compared to standard of care. Further data are needed on the efficacy and effectiveness of LPVr for severe COVID19. An update to this dynamic benefit-risk assessment will be published when sufficient data are made available from further peer-reviewed studies that impact significantly on the benefit-risk of LPVr.

\subsection{Strengths and Limitations}

A strength of this approach is the inclusion of all key benefits and risks in the same model and a transparent framework into which further data can be included as and when these become available. The method has a significant advantage compared with systematic reviews, which are equally comprehensive but focus only on efficacy or safety, rather than considering both together. When sufficient data are available, the methodology allows benefits and risks to be ranked, and weightings applied based on this ranking, with further quantitative analysis. The reproducibility of this assessment allows multiple treatments to be assessed using this approach, thereby allowing direct comparison between different treatments. This is of great significance during the current COVID-19 crisis, in which several potential interventions currently under evaluation need to be assessed and evaluated in real time, and where new data need to be incorporated quickly. Regulatory decision makers are also familiar with this framework, facilitating interpretation.

A limitation of the benefit-risk assessment presented at this time is the relative paucity of data that has been included in the model, as many clinical trials assessing LPVr are still ongoing. In addition, trials for which data were available had very small sample sizes and were likely to be underpowered when examining non-primary outcomes. Causality cannot be confirmed for many outcomes at this point in time but in general there is a need for further data regarding outcomes in COVID-19. It is anticipated that such data will become available in due course and allow us to comment further on the causal association between LPVr and outcomes included in the value tree. The dynamic nature of this benefit-risk assessment allows further updates in the future if sufficient data are made available to significantly impact the conclusion of the benefit-risk assessment.

Study quality was not considered in the assessment, although we only included peer-reviewed articles. The importance of each benefit and risk was assessed by two physicians and ranked accordingly based on their clinical judgement. Because of the dynamic nature of the benefit-risk assessment, other experts could be engaged in the future to reassess the importance of the benefits and risks and revise the value tree. Outcomes displayed in the value tree were identified from a variety of sources and may therefore be related to different underlying diseases with different management including potentially different doses of LPVr. Efficacy outcomes could also be dependent on the time of administration of LPVr. Further, while we defined standard of care as no use of a specific pharmaceutical treatment for COVID-19 but provision of standard supportive therapy within the hospital setting, we acknowledge that standard of care may vary by setting and country.

\section{Conclusions}

Based on currently available data, there was no clear benefit for use of LPVr compared to standard of care in severe COVID-19. Risk data suggested a possible decrease in serious adverse events. There was a reduction in ARDS with LPVr in one study. Overall, the benefit-risk profile for LPVr in severe COVID-19 cannot be considered positive until further efficacy and effectiveness data become available.

\section{Declarations}

Author contributions VO assisted with the study design and data extraction and wrote the study proposal and the first draft of the manuscript. MD assisted with study design, identified outcomes of interest, constructed the value tree and assisted with data extraction. SD assisted with the value tree and rankings. DR, SL, JD and AE assisted with the study design, literature searching and data extraction. SAWS assisted with the concept, study design and manuscript revisions. All authors reviewed the manuscript, contributed to revisions, and approved the manuscript and accept full responsibility for its overall content.

Funding The Drug Safety Research Unit (DSRU) is an independent academic institution that works in association with the University of Portsmouth. No funding was received for this project.

Data Availability Data used in this analysis are available from the references supplied.

Conflict of Interest The DSRU is an independent charity (No. 327206) that works in association with the University of Portsmouth. It receives unconditional donations from pharmaceutical companies. The companies have no control on the conduct or the publication of the studies conducted by the DSRU. AbbVie, the marketing authorisation holder for lopinavir-ritonavir, has paid the DSRU for consultancy services unrelated to lopinavir-ritonavir. AbbVie is also providing support for an unrelated methodological project led by the DSRU as a part of a large group of pharmaceutical companies, unrelated to lopinavir-ritonavir or any other AbbVie product. Saad Shakir is a member of the Data Safety Monitoring Board for Diurnal and Biogen. Miranda Davies, Vicki Osborne, Samantha Lane, Debabrata Roy, Sandeep Dhanda, Jacqueline Denyer, Alison Evans and Saad Shakir have no other conflicts of interest to declare.

Ethics Approval This study was conducted in accordance with international ethical guidelines. Ethics approval was not required for this study. 
Consent to participate Not applicable.

Consent for publication Not applicable.

Code availability (software application or custom code) Not applicable.

Open Access This article is licensed under a Creative Commons Attribution-NonCommercial 4.0 International License, which permits any non-commercial use, sharing, adaptation, distribution and reproduction in any medium or format, as long as you give appropriate credit to the original author(s) and the source, provide a link to the Creative Commons licence, and indicate if changes were made. The images or other third party material in this article are included in the article's Creative Commons licence, unless indicated otherwise in a credit line to the material. If material is not included in the article's Creative Commons licence and your intended use is not permitted by statutory regulation or exceeds the permitted use, you will need to obtain permission directly from the copyright holder. To view a copy of this licence, visit http://creativecommons.org/licenses/by-nc/4.0/.

\section{References}

1. Cui J, Li F, Shi ZL. Origin and evolution of pathogenic coronaviruses. Nat Rev Microbiol. 2019;17(3):181-92. https://doi. org/10.1038/s41579-018-0118-9.

2. Wang C, Horby PW, Hayden FG, Gao GF. A novel coronavirus outbreak of global health concern. Lancet. 2020;395(10223):470 3. https://doi.org/10.1016/S0140-6736(20)30185-9.

3. WHO. Naming the coronavirus disease (COVID-19) and the virus that causes it. Geneva: WHO; 2020.

4. WHO. WHO announces COVID-19 outbreak a pandemic. Geneva: WHO; 2020.

5. Chen N, Zhou M, Dong X, Qu J, Gong F, Han Y, et al. Epidemiological and clinical characteristics of 99 cases of 2019 novel coronavirus pneumonia in Wuhan, China: a descriptive study. Lancet. 2020;395(10223):507-13. https://doi.org/10.1016/S0140 $-6736(20) 30211-7$.

6. Croxtall JD, Perry CM. Lopinavir/ritonavir: a review of its use in the management of HIV-1 infection. Drugs. 2010;70(14):1885915. https://doi.org/10.2165/11204950-000000000-00000.

7. Chu CM, Cheng VCC, Hung IFN, Wong MML, Chan KH, Chan $\mathrm{KS}$, et al. Role of lopinavir/ritonavir in the treatment of SARS: initial virological and clinical findings. Thorax. 2004;59(3):252-6. https://doi.org/10.1136/thorax.2003.012658.

8. University of Oxford. Randomised Evaluation of COVID-19 Therapy (RECOVERY) trial. 2020. https://www.recoverytrial. net. Accessed 22 June 2020.

9. WHO. The (Norwegian) NOR solidarity multicenter trial on the efficacy of different anti-viral drugs in SARS-CoV-2 infected patients. Geneva: WHO; 2020.

10. WHO. A multi-centre, adaptive, randomized, open-label, controlled clinical trial of the safety and efficacy of investigational therapeutics for the treatment of COVID-19 in hospitalized patients (CATCO: Canadian Treatments for COVID-19), in conjunction with the public health emergency SOLIDARITY trial (World Health Organization). Geneva: WHO; 2020.

11. Dorward J, Gbinigie K. Lopinavir/ritonavir: a rapid review of effectiveness in COVID-19. Oxford: Centre for Evidence Based Medicine, University of Oxford; 2020. https://www.cebm.net/ covid-19/lopinavir-ritonavir-a-rapid-review-of-the-evidence-foreffectiveness-in-treating-covid/.

12. Ford N, Vitoria M, Rangaraj A, Norris SL, Calmy A, Doherty M. Systematic review of the efficacy and safety of antiretroviral drugs against SARS, MERS or COVID-19: initial assessment. J Int AIDS Soc. 2020;23(4):e25489. https://doi.org/10.1002/ jia2.25489.

13. Osborne V, Davies M, Roy D, Tescione F, Shakir SAW. Systematic benefit-risk assessment for buprenorphine implant: a semiquantitative method to support risk management. BMJ Evid Based Med. 2020. https://doi.org/10.1136/bmjebm-2019-111295.

14. PROTECT. PROTECT benefit-risk. 2015. http://protectbenefitr isk.eu/. Accessed 22 June 2020.

15. Nixon R, Dierig C, Mt-Isa S, Stockert I, Tong T, Kuhls S, et al. A case study using the PrOACT-URL and BRAT frameworks for structured benefit risk assessment. Biom J. 2016;58(1):8-27. https ://doi.org/10.1002/bimj.201300248.

16. Davies M, Osborne V, Lane S, Roy D, Dhanda S, Evans A, et al. Remdesivir in treatment of COVID-19: a systematic benefit-risk assessment. Drug Saf. 2020. https://doi.org/10.1007/s40264-02000952-1.

17. PROTECT. BRAT (Benefit-Risk Action Team). 2015. https:// protectbenefitrisk.eu/BRAT.html. Accessed 16 Jun 2020.

18. Cao B, Wang Y, Wen D, Liu W, Wang J, Fan G, et al. A trial of lopinavir-ritonavir in adults hospitalized with severe Covid-19. N Engl J Med. 2020;382(19):1787-99. https://doi.org/10.1056/ NEJMoa2001282.

19. Giudicessi JR, Noseworthy PA, Friedman PA, Ackerman MJ. Urgent guidance for navigating and circumventing the QTcprolonging and Torsadogenic potential of possible pharmacotherapies for Coronavirus Disease 19 (COVID-19). Mayo Clin Proc. 2020;95(6):1213-21. https://doi.org/10.1016/j.mayoc p.2020.03.024.

20. Guzik TJ, Mohiddin SA, Dimarco A, Patel V, Savvatis K, MarelliBerg FM, et al. COVID-19 and the cardiovascular system: implications for risk assessment, diagnosis, and treatment options. Cardiovasc Res. 2020. https://doi.org/10.1093/cvr/cvaa106.

21. Naksuk N, Lazar S, Peeraphatdit TB. Cardiac safety of off-label COVID-19 drug therapy: a review and proposed monitoring protocol. Eur Heart J Acute Cardiovasc Care. 2020;9(3):215-21. https://doi.org/10.1177/2048872620922784.

22. Sapp JL, Alqarawi W, MacIntyre CJ, Tadros R, Steinberg C, Roberts JD, et al. Guidance on minimizing risk of drug-induced ventricular arrhythmia during treatment of COVID-19: a statement from the Canadian Heart Rhythm Society. Can J Cardiol. 2020. https://doi.org/10.1016/j.cjca.2020.04.003.

23. AbbVie Ltd. Kaletra summary of product characteristics. 2020. https://www.medicines.org.uk/emc/product/221. Accessed 22 June 2020.

24. Testa S, Prandoni P, Paoletti O, Morandini R, Tala M, Dellanoce $\mathrm{C}$, et al. Direct oral anticoagulant plasma levels striking increase in severe COVID-19 respiratory syndrome patients treated with antiviral agents: the Cremona experience. J Thromb Haemost. 2020. https://doi.org/10.1111/jth.14871.

25. Ning L, Liu L, Li W, Liu H, Wang J, Yao Z, et al. Novel coronavirus (SARS-CoV-2) infection in a renal transplant recipient: case report. Am J Transpl. 2020. https://doi.org/10.1111/ajt.15897.

26. Wu C, Chen X, Cai Y, Xia J, Zhou X, Xu S, et al. Risk factors associated with acute respiratory distress syndrome and death in patients with coronavirus disease 2019 pneumonia in Wuhan, China. Intern Med. 2019. https://doi.org/10.1001/jamaintern med.2020.0994.

27. Tsang KWT, Ooi GC, Ho PL. Diagnosis and pharmacotherapy of severe acute respiratory syndrome: what have we learn? Eur Respir J. 2004;24(6):1025-32. https://doi.org/10.1183/09031 936.04.00092004.

28. Pal R, Bhadada SK. COVID-19 and diabetes mellitus: an unholy interaction of two pandemics. Diabetes Metab Syndr. 2020;14(4):513-7. https://doi.org/10.1016/j.dsx.2020.04.049. 
29. Rubel AR, Chong PL, Abdullah MS, Asli R, Momin RN, Mani $\mathrm{BI}$, et al. Letter to the Editor. Lipemic serum in patients with COVID-19 undergoing treatment. J Med Virol. 2020. https://doi. org/10.1002/jmv.25942.

30. Sun J, Deng X, Chen X, Huang J, Huang S, Li Y, et al. Incidence of adverse drug reactions in COVID-19 patients in China: an active monitoring study by hospital pharmacovigilance system. Clin Pharmacol Ther. 2020. https://doi.org/10.1002/cpt.1866.

31. Wang L, Xu X, Ruan J, Lin S, Jiang J, Ye H. Quadruple therapy for asymptomatic COVID-19 infection patients. Expert Rev Anti Infect Ther. 2020;3:1-8. https://doi.org/10.1080/14787 210.2020.1758066.

32. Fan Z, Chen L, Li J, Cheng X, Jingmao Y, Tian C, et al. Clinical features of COVID-19-related liver damage. Clin Gastroenterol Hepatol. 2020. https://doi.org/10.1016/j.cgh.2020.04.002.

33. Cai Q, Huang D, Yu H, Zhu Z, Xia Z, Su Y, et al. Characteristics of liver tests in COVID-19 patients. J Hepatol. 2020. https://doi. org/10.1016/j.jhep.2020.04.006.

34. Cai Q, Yang M, Liu D, Chen J, Shu D, Xia J, et al. Experimental treatment with favipiravir for COVID-19: an open-label control study. Engineering (Beijing). 2020. https://doi.org/10.1016/j. eng.2020.03.007.
35. Huang M, Tang T, Pang P, Li M, Ma R, Lu J, et al. Treating COVID-19 with chloroquine. J Mol Cell Biol. 2020;12(4):322-5. https://doi.org/10.1093/jmcb/mjaa014.

36. Li Y, Xie Z, Lin W, Cai W, Wen C, Guan Y, et al. Efficacy and safety of lopinavir/ritonavir or arbidol in adult patients with mild/ moderate COVID-19: an exploratory randomized controlled trial. Med. 2020. https://doi.org/10.1016/j.medj.2020.04.001.

37. Ye XT, Luo YL, Xia SC, Sun QF, Ding JG, Zhou Y, et al. Clinical efficacy of lopinavir/ritonavir in the treatment of Coronavirus disease 2019. Eur Rev Med Pharmacol Sci. 2020;24(6):3390-6. https://doi.org/10.26355/eurrev_202003_20706.

38. Zhu Z, Lu Z, Xu T, Chen C, Yang G, Zha T, et al. Arbidol monotherapy is superior to lopinavir/ritonavir in treating COVID19. J Infect. 2020;81(1):e21-e2323. https://doi.org/10.1016/j. jinf.2020.03.060.

39. Wen CY, Xie ZW, Li YP, Deng XL, Chen XT, Cao Y, et al. Realworld efficacy and safety of lopinavir/ritonavir and arbidol in treating with COVID-19: an observational cohort study. Zhonghua nei ke za zhi. 2020;59:E012. https://doi.org/10.3760/cma.j.cn112 138-20200227-00147. 